\title{
Análisis jurídico de la responsabilidad civil extracontractual derivada del daño ambiental por la producción de ladrillos en Jauja, Junín
}

\author{
Legal analysis of the tort civil liability for environmental damage by the \\ production of bricks in Jauja, Junín
}

\author{
Jonathan Betalleluz Montero ${ }^{1,}$, , Cristhian Mamani Romero ${ }^{1}$, Robert Gutiérrez León ${ }^{1}$, Emma C. Jaramillo Cabrera ${ }^{1}$ \\ ${ }^{1}$ Universidad Continental - Perú, Facultad de Derecho
}

\section{RESUMEN}

El presente trabajo de investigación ha sido motivado por la actividad ladrillera artesanal que produce impactos en el ecosistema rural del distrito de Huertas, provincia de Jauja, departamento de Junín. El daño ambiental de dicha actividad constituye la vulneración de un conjunto de derechos fundamentales (salud, vida, libertad, etc.), generándose así un daño patrimonial y no patrimonial, colectivo, ya que consideramos de suma importancia hacer un análisis sobre la regulación de la responsabilidad civil extracontractual por daño ambiental que deriva de dicha actividad. Es así que se pudo obtener información documental que permitió ver la gravedad en la cual se encontraba la localidad de Huertas, ya que la contaminación generada por la cocción de ladrillos causaba perjuicio no solo en la salud de los pobladores, sino que éstos estaban expuestos a asumir una posible responsabilidad derivada por dicha actividad contaminante. Luego, se pudo analizar la posibilidad de regulación de la materia extracontractual tomando como referencia a los principios de precaución y prevención, netos del campo del Derecho Ambiental, ya que estos coadyuvaran a una mejor regulación normativa frente a los vacíos legales referente a los factores de atribución, nexo causal y los sujetos responsables. A manera de conclusión se puede determinar que el fenómeno de contaminación es un tema de suma importancia en el contexto local, nacional e internacional.

Palabras clave: Contaminación ambiental, responsabilidad civil por daño ambiental, responsabilidad civil extracontractual, industria ladrillera.

\section{ABSTRACT}

This research work has been motivated by activity handmade brick that produces impacts on the rural ecosystem of the ecosystem of the district of Huertas, province of Jauja, Junín department. The environmental damage of such activity constitutes infringement of a set of fundamental rights (health, life, freedom, etc.), generating thus a patrimonial damage and non-equity, collective, since we consider of utmost importance make an analysis on the regulation of non-contractual civil liability for environmental damage resulting from such activity. So that could get documentary information allowing to see the gravity which was the town of Huertas, since the pollution generated by bricks caused not only perjudice the heatlh of residents, but these were exposed to assume a possible liability for the polluting activity. Then, you could analyze the possibility of regulation of the matter in tort by reference to the principles of precaution and prevention, only in the field of environmental law, since these back to a better normative regulation against loopholes concerning attribution factors, causal link and responsable subjects. A conclusion can be determined that the phenomenon of pollution is an issue of utmost importance in the context of local, national and international.

Keywords: Environmental pollution, civil liability for environmental damage, non-contractual civil liability, brick industry.

Historial del artículo:

Recibido, 17 de setiembre de 2017; aceptado, 2 de noviembre de 2017; disponible en línea, 05 de enero de 2018

* Estudiante de la facultad de Derecho de la Universidad Continental.

Correo: jbetalleluz23@gmail.com 


\section{INTRODUCCIÓN}

La conservación del medio ambiente como principio jurídico rector se recoge en la Constitución Política del Perú, que en su numeral 22 del artículo $2^{\circ}$, Capítulo I dedicado a los Derechos Fundamentales de la Persona, reconoce "el derecho de toda persona a gozar de un medio ambiente equilibrado y adecuado al desarrollo de su vida".

La fabricación artesanal de ladrillos es considerada una de las principales fuentes de emisión de contaminantes a la atmosfera, caracterizándose por una variedad muy amplia de tipos y cantidades de combustibles utilizados en su etapa final de producción. En dicho contexto encontramos que en el Distrito de Huertas Jauja, se viene realizando dicha actividad por pequeñas empresas familiares informales, actividad que se inicia
Este hecho nos permitió determinar que toda actividad, a pesar de ser artesanal, genera riesgos de contaminación ambiental, sin la necesidad de la existencia de una conducta dolosa por parte del actor.

Por lo expuesto, se formuló las siguientes interrogantes:

1. ¿Qué rol debe desempeñar el sistema de responsabilidad civil frente a los daños ambientales en los casos de contaminación por quema de ladrillos?

2. ¿̇La normativa peruana brinda una adecuada tutela frente a los daños ambientales?

3. ¿Cuál es el problema en la identificación de los responsables?

4. ¿̇La naturaleza de la responsabilidad civil por daño ambiental es netamente objetiva?

$\mathrm{Y}$, el presente trabajo tuvo por finalidad dar algunos

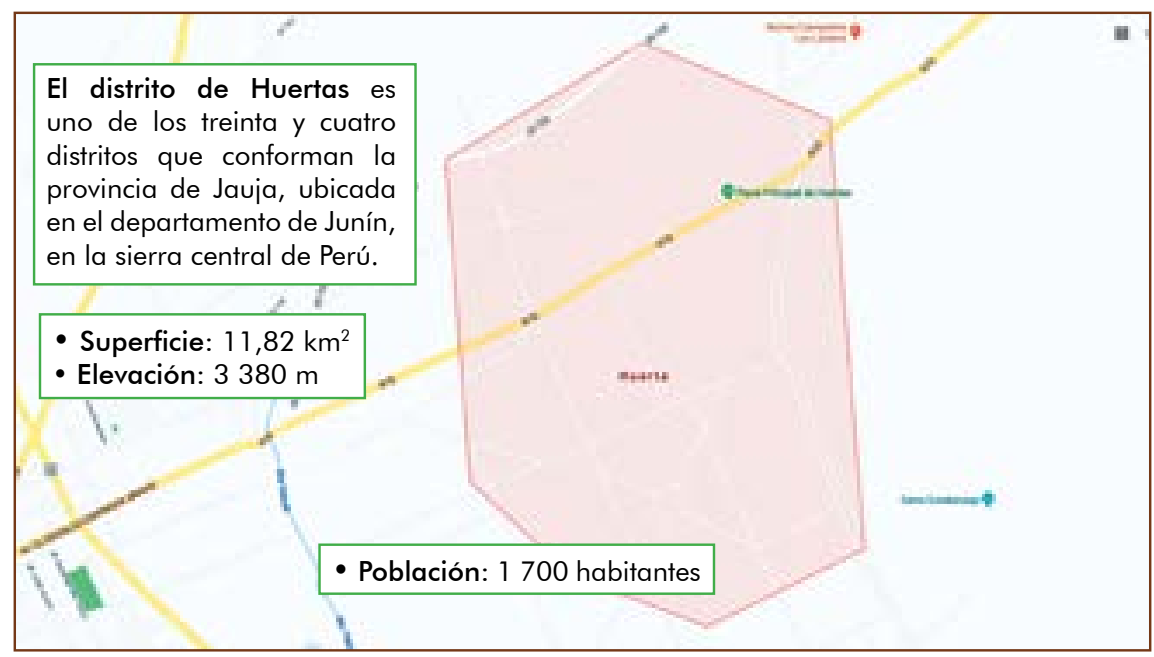

Figura 1. Ubicación geográfica del distrito de Huertas - Jauja.

Fuente: GOOGLE MAPS - 2017.

con la extracción de la arcilla, manipulación, mezclado y finalmente se culmina con el secado de los ladrillos en hornos artesanales, utilizando para su cocción insumos químicos (combustible, plásticos y llantas), exponiendo así la salud de los propios pobladores, y causando a su vez un daño irreversible al medio ambiente.

Para la presente investigación, se consultaron diversas fuentes bibliográficas sobre la Responsabilidad Civil derivada del daño ambiental, teniendo en consideración que la legislación peruana no ha efectuado investigaciones arduas sobre el rol que debe desempeñar la responsabilidad civil, ello frente a la existencia de los principios ambientales de prevención y precaución. Asimismo, se utilizó diversas fuentes de información como entrevistas a los propios pobladores, colindantes, funcionarios y servidores públicos de las Municipalidad de Huertas y Jauja respectivamente. alcances sobre el análisis jurídico de la responsabilidad civil extracontractual que deriva de la producción artesanal de ladrillos.

\section{MATERIAL Y MÉTODOS}

En el presente caso se utilizó el método hipotéticodeductivo por acumulación de datos empíricos, generando como principal resultado, que la regulación normativa sobre la responsabilidad civil extracontractual derivada de daño ambiental en el Código Civil es casi nula, y la que se regula en la Ley General del Ambiente, es confusa; en consecuencia, se encontró una regulación inadecuada que brinde una protección efectiva frente al daño ambiental que se deriva de la actividad artesanal de producción de ladrillos. 
Asimismo se utilizó el método analítico-observacional que permitió recabar información documental sobre el problema ambiental suscitado en la localidad de Huertas.

Huertas está ubicado a $5 \mathrm{~km}$ al Este de la Provincia de jauja (figura 1) departamento de Junín y tiene una gran variedad en atractivos turísticos una de ellas es este majestuoso Templo de los Yogas o la Gran Fraternidad Universal de los Yogas que está ubicado al este de la plaza del Huertas a una distancia de $1 \mathrm{~km}$ en el Barrio Coriac, al igual que la Portada Colonial en el barrio Rumichaca, un mirador espectacular ubicado en el Barrio Manzana Cucho Pomate de donde se puede apreciar todo el Valle del Mantaro y la laguna de Paca al mismo tiempo podemos informar que en el Distrito de Huertas se fabrican las mejores tejas y ladrillos que son repartidos a nivel nacional y estas fábricas están ubicados en el Barrio de Condorsinja en donde se elementos y compuestos provocan irritación a la piel, ojos y membranas mucosas, trastornos en las vías respiratorias, en el sistema nervioso central, depresión y eventualmente cáncer; características que los hacen inaceptables para ser utilizados para este fin (Benites, 2013).

La gran mayoría de ladrilleras realizan la fabricación de ladrillos en su entorno familiar, presentando así un alto grado de informalidad y utilizando técnicas artesanales para la fabricación de sus productos. La planta de fabricación está representada básicamente por el horno y un espacio de terreno como patio de labranza. Las ladrilleras artesanales emplean hornos fijos de fuego directo y de techo abierto (figura 2).

En ese sentido, el proceso de la producción artesanal, se torna ya no como un proceso inofensivo debido a los diversos insumos utilizados para su elaboración, ya

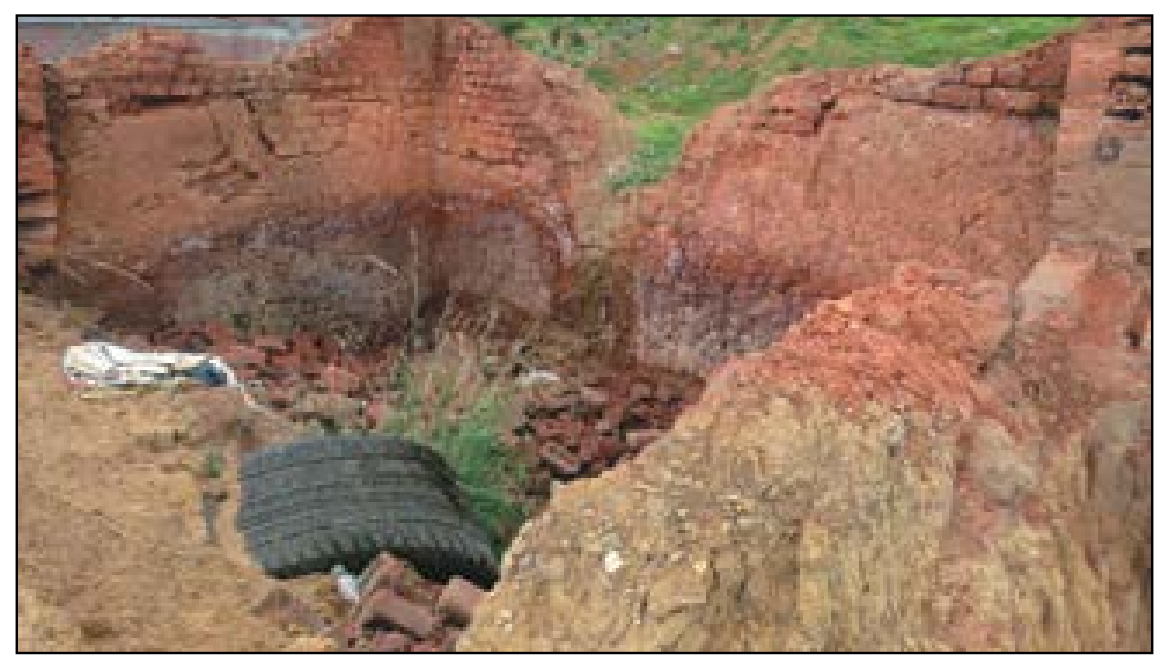

Figura 2. Quema de llantas en hornos para la cocción de ladrillos en Huertas-Jauja (2017).

puede apreciar el cerro en forma de este apreciado ave andino que es el Cóndor que tiene relación con el Indio dormido de la Laguna de Paca (Enperu, 2017).

La fabricación de ladrillos artesanales de arcilla cocida, se ha convertido en un problema ecológico en muchas ciudades de nuestro país, éste es el caso de las ladrilleras ubicadas en el Distrito de Huertas, debido al tipo de combustibles que se utilizan para la cocción de dichos productos, que al ser sometidos a combustión, emiten una gran cantidad de gases a la atmósfera.

El uso de estos materiales como combustible genera emisiones de gases altamente tóxicos y cancerígenos como óxidos de azufre (SOx), óxidos de nitrógeno (NOx), compuestos orgánicos volátiles (COV), hidrocarburos aromáticos poli nucleares, dioxinas, furanos, benceno, bifenilos poli clorados y metales pesados como $\mathrm{As}, \mathrm{Cd}, \mathrm{Ni}, \mathrm{Zn}, \mathrm{Hg}, \mathrm{Cr}$, $\mathrm{V}$, etc. estos que el problema medular se genera con el proceso de cocción de los ladrillos, donde se genera una densa humareda de color negro que contiene partículas en suspensión y compuestos orgánicos volátiles.

\section{RESULTADOS}

La tabla 2 muestra las causas y consecuencias de esta actividad, las mismas que tienen una relación descriptiva que centra la problemática del por qué se persiste en la realización de esta actividad.

Se determinó que, esta actividad significa para estas familias parte de su desarrollo económico, conocimientos que han sido heredados por parte de sus ancestros de manera empírica. Por ello, ignoran los límites y consecuencias de sus actos, es decir, de la 
responsabilidad civil extracontractual que generan al realizar dicha actividad, debido a que si bien no tienen la intención de dañar, lo hacen de manera directa (al medio ambiente) e indirecta (la salud pública).

Dentro del distrito de Huertas se pudo recoger testimonios de vecinos que se encuentran incomodos con la actividad de quema de llantas que realizan las ladrilleras artesanales, ya que los humos viajan a años de edad. Así mismo la señora señaló haber sido afectada por algunos problemas respiratorios desde hace seis años atrás" (Betalleluz Montero, J. Gutiérrrez León, R. Mamani Romero, C.U., 2017).

Asimismo, se pudo recoger el testimonio de Juan, un ladrillero de la zona de Cormes, Jauja, quien señala que: "Todas las ladrilleras en Huertas son informales y solo algunos ladrilleras son aparentemente formales.

Tabla 1

Generación de contaminantes según la etapa del proceso de producción de ladrillos artesanales.

\begin{tabular}{|c|c|c|c|}
\hline $\mathrm{N}^{\circ}$ & Etapas & $\begin{array}{c}\text { Actividades que } \\
\text { generan contaminantes }\end{array}$ & Tipos de contaminantes \\
\hline 01 & $\begin{array}{l}\text { Extracción de la } \\
\text { arcilla y tierra }\end{array}$ & Extracción con herramientas manuales. & $\begin{array}{l}\text { Cambios en la morfología del } \\
\text { terreno. }\end{array}$ \\
\hline 02 & Mezclado & Extracción con maquinaria pesada. & $\begin{array}{l}\text { Cambios en la morfología del } \\
\text { terreno y uso de la tierra agrícola. }\end{array}$ \\
\hline 03 & Moldeado & No genera contaminantes. & Ninguno. \\
\hline 04 & Secado & $\begin{array}{l}\text { Durante el secado solo se desprende vapor } \\
\text { de agua. }\end{array}$ & Ninguno. \\
\hline 05 & Carga del Horno & $\begin{array}{l}\text { Resuspende partículas del suelo y de la } \\
\text { fricción de los mismo ladrillos. }\end{array}$ & Partículas en suspensión. \\
\hline 06 & Cocción & $\begin{array}{l}\text { Uso de combustible en la cocción de ladrillos: } \\
\text { llantas, aserrín, madera, plásticos, etc. }\end{array}$ & $\begin{array}{l}\text { Partículas en suspensión: } \mathrm{SO} 2, \mathrm{NO} 2 \text {, } \\
\mathrm{CO} \text { y compuestos orgánicos } \\
\text { volátiles. }\end{array}$ \\
\hline 07 & Clasificación & $\begin{array}{l}\text { Descarte de productos rotos, fisurados y mal } \\
\text { cocidos. }\end{array}$ & Residuos sólidos inertes. \\
\hline 08 & Despacho & Descarte de productos rotos. & Residuos sólidos inertes. \\
\hline
\end{tabular}

Fuente: Elaboración propia

Tabla 2

Relación de las causas y consecuencias de la producción de los ladrillos artesanales.

\begin{tabular}{|c|c|}
\hline Causas & Consecuencias \\
\hline $\begin{array}{l}\text { 1. Único sustento de vida: Fuente } \\
\text { generadora de trabajo, considerado } \\
\text { como el principal mercado proveedor. }\end{array}$ & $\begin{array}{l}\text { Ambientales: degradación del suelo, } \\
\text { contaminación del aire y agua. } \\
\text { Salud: múltiples enfermedades respiratorias }\end{array}$ \\
\hline $\begin{array}{l}\text { 2. Socio-culturales: conocimientos empíricos } \\
\text { heredados, Falta de educación ambiental. }\end{array}$ & y de la piel \\
\hline $\begin{array}{l}\text { 3. Políticas: Desinterés de las autoridades, } \\
\text { falta de políticas de desarrollo medio } \\
\text { ambiental. }\end{array}$ & $\begin{array}{l}\text { Informalidad: evasión tributaria, pésimas } \\
\text { condiciones laborales }\end{array}$ \\
\hline
\end{tabular}

Fuente: Elaboración propia

través del aire y las cenizas se van esparciendo, con lo que se genera la contaminación del aire. De manera indirecta, conlleva consecuencias en la salud de las personas expuestas o que residen cerca a las ladrilleras, afectando principalmente las vías respiratorias, como es el caso de caso de Timotea que mediante una entrevista manifestó que, "a consecuencia de los humos producidos por los hornos su nieta, de trece años sufre problemas respiratorios desde los seis
Asimismo menciona que, "en la zona de Cormes se usa el carbón de mina para calentar el horno pero en las ladrilleras de Condorcinga solo hacen uso de las llantas como insumo de combustión" (Betalleluz Montero, J. Gutiérrrez León, R. Mamani Romero, C.U., 2017).

Respecto al marco jurídico legal aplicable, se determinó que existen diversas normas como la Constitución 
Política del Perú que establece de forma explícita los derechos fundamentales de las personas como el derecho a la vida y a la salud, en concordancia con otras como: la Ley General de Salud - Ley $N^{\circ}$ 26842 (que considera a la salud como condición indispensable del desarrollo humano, así considerada también como el deber del estado de regularla, vigilar y promoverla), la Ley General del Ambiente - Ley $N^{\circ} 28611$ (que adopta políticas de desarrollo sostenible del país en armonía con el mejoramiento de vida, mediante la protección, conservación y recuperación del ambiente), la Ley Orgánica para el Aprovechamiento Sostenible de los Recursos Naturales - Ley N 26821 (que regula el aprovechamiento o utilización de los recursos naturales renovables y no renovables, de forma tal que se realice de la manera permitida de acuerdo la capacidad medioambiental), el Código Civil (que regula el derecho de propiedad, así como la institución de la responsabilidad civil), el Código Penal (que reconoce los delitos contra los recursos naturales y el medio ambiente).

Sin embargo, estas normas son transgredidas por parte de los agentes contaminadores con debido a su desconocimiento normativo y su falta de cultura ambiental.

\section{DISCUSIÓN}

Atrajo el interés del grupo para investigar y analizar esta problemática ambiental desde la óptica del derecho, que existe un marco normativo pero también vacíos legales, a ello se agrega la inaplicación de las normas por parte de las autoridades situación que se agrava por el desinterés por el tema ambiental, así como la explotación indiscriminada de los recursos naturales renovables y no renovables (arcilla, tala de árboles, agua).

La producción artesanal de los ladrillos trae consigo el riesgo no solo de la modificación panorámica o de la naturaleza que genera la extinción o migración de la biodiversidad, en efecto, hecho que pone en juego también la salud pública.

Sin embargo, se reconoció que una intervención drástica podría resultar desacertada, ya que se afectaría económicamente estos negocios familiares informales lo que llevó a pensar al grupo que una intervención así requeriría de una estrategia que comprenda en principio la formulación de un plan que incluya:

- Políticas para la regulación, formalización y creación de asociaciones de las empresas ladrilleras.

- Constantes capacitaciones profesionales en el nivel técnico como legal.

- Ayuda financiera para la mejora el acceso de nuevas tecnologías para la mejora del proceso de producción.

- Reubicación de la empresas para la recuperación y habilitación de espacios

- Saneamiento ambiental.

Por otro lado, cuando hacemos referencia en cuanto al tema de la contaminación ambiental y en la tarea de poder determinar la responsabilidad ya sea civil, penal o administrativa, entendemos que ésta, debe ser vista desde dos dimensiones: por un lado, se encuentra el aspecto material o patrimonial (como la degradación del suelo, aire y agua), y por otro el aspecto formal o no patrimonial (como la violación de derechos fundamentales e inherentes a la persona humana, como consecuencia de la contaminación, por ejemplo: la vida, la dignidad de la persona, etc.). Puesto que, ambos aspectos tiene vinculo respecto del hecho causante del daño y la población involucrada tal como se muestra en la tabla 2.

Lo expuesto se fundamenta en la forma del proceso de producción: primero por el uso indiscriminado de los diversos insumos para la elaboración y segundo porque se genera una contaminación con repercusiones graves e irreversibles, que afecta directamente no solo la flora y fauna sino que pone en peligro la "salud pública" teniendo efectos a nivel local pero también global dada su característica de "actividad constante". Por ende, cabe precisar que no solo se trata de un simple problema ecológico dado que se está violando toda una gama de derechos fundamentales. Por ello "este tipo de daño afecta directamente al medio ambiente e indirectamente a las personas individualmente consideradas, en su salud o su patrimonio" (Carhuatocto Sandoval, 2007). Asimismo, para resaltar Amelia del Castillo (2017) señala que, "En el primer caso el bien jurídico afectado es el medio ambiente y en el segundo es específicamente la persona y su patrimonio".

En concreto la Ley General del Ambiente 28611, crea un espacio armónico y propicio de manera que pretende mediar el dialogo o las relaciones sociales de individuos o grupos sociales en caso de surgimiento de conflictos socio-económicos referidos a temas de contaminación ambiental, es decir, que lo que busca es el bienestar y la mejora de la calidad de vida de la población. De manera que la LGA, establece los mecanismos de Conciliación y Arbitraje entre otros como medios de resolución y gestión de conflictos ambientales para la determinación de la responsabilidad civil por daños ambientales "siempre y cuando se trate de derechos de libre disposición".

Así mismo, cabe resaltar que, a lo largo de la investigación no se ha podido recabar información o estudios previos que nos pudieran servir como 
antecedente acerca del tema, es decir que solo hay algunos trabajos que si bien tratan sobre la precariedad con la que se ejecutan estos procesos, los niveles de contaminación que se generan y propuestas de mejoras para la producción; pero dejan de lado el ámbito preocupante que es el análisis del derecho, su aplicación así como la afectación y la percepción de la población involucrada en la problemática ambiental.

\section{La responsabilidad civil por daño ambiental}

\section{La responsabilidad civil en la legislación peruana}

En la legislación peruana se regula la responsabilidad civil referida al aspecto de indemnizar los daños que deriven de:

- Incumplimiento de una obligación voluntaria contenida en un contrato (responsabilidad civil contractual), o

- El resultado de una conducta que cause un daño a otra persona sin existir vinculo obligacional alguno, mas solo se vulnera el deber jurídico general de no dañar a otro (responsabilidad extracontractual).

Asimismo Taboada Córdova (2000) señala que existen cuatros requisitos para poder determinar la responsabilidad civil, los cuales son: la antijuricidad, el daño causado, la relación de causalidad y los factores de atribución.

- La antijuricidad: La conducta antijurídica, se da cuando una conducta contraviene una norma prohibitiva.

- Daño causado: Se entiende al daño como aquella lesión a todo derecho subjetivo que se encuentra protegido por el ordenamiento jurídico.

- La relación de causalidad: Es la relación de causaefecto entre la conducta típica o atípica y el daño producido a la víctima, si no existiese relación de causa-efecto o no se pudiese probar, no existirá ningún tipo de responsabilidad.

- Factores de atribución: El factor de atribución depende del tipo de responsabilidad a la cual nos enfrentemos, así tenemos que en la responsabilidad civil contractual, existe el factor subjetivo, que vendría a ser la culpa, en sus tres grados (leve, grave o inexcusable y dolo), mientras que en la responsabilidad civil extracontractual, tenemos al factor objetivo mediante el cual sólo se debe probar fehacientemente que la conducta que ha causado el daño es peligrosa o riesgosa, sin necesidad de acreditar la culpabilidad.

Finalmente, es prudente dar una definición de daño ambiental, entendiendo a éste como aquel desgaste de los recursos que se produce a consecuencia de la contaminación y degradación ambiental en los procesos de producción, distribución o comercialización (Andía Chávez, 2009).

\section{La aplicación de la responsabilidad civil a los daños ambientales}

Para poder encaminarse hacia un mismo resultado, se indica que la responsabilidad que deriva de daños ambientales, es una responsabilidad extracontractual, entonces, se puede decir que cuando una actividad ejercida por persona natural o jurídica que represente una fuente de provecho para ésta y un riesgo adicional y extraordinario para el resto, ya sea personas o recursos naturales, conlleva a un resarcimiento de daños y perjuicios que se configura por la utilidad que perciben los agentes de la actividad contaminante.

Es así que, el artículo IV del TP de la Ley General del Ambiente establece que: "Toda persona tiene el derecho a una acción rápida, sencilla y efectiva, ante las entidades administrativas y jurisdiccionales, en defensa del ambiente y de sus componentes, velando por la debida protección de la salud de las personas en forma individual y colectiva, la conservación de la diversidad bilógica, el aprovechamiento sostenible de los rgecurso naturales, así como la conservación del patrimonio cultural vinculado a aquellos".

Entonces podemos determinar que, en el Perú existen normas de carácter sustantivo y adjetivo que establecen la legitimidad para obrar respecto al daño ambiental generado, es el caso del artículo $82^{\circ}$ del Código Procesal Civil que señala quienes son las personas y entidades facultadas para promover $\circ$ intervenir en procesos de vulneración de intereses difusos, lo mismo hace el Artículo $143^{\circ}$ de la Ley General del Ambiente que señala que, "Cualquier persona, natural o jurídica, está legitimada para ejercer la acción a que se refiere la presente Ley, contra quienes ocasionen o contribuyen a ocasionar un daño ambiental". Sin embargo, nace la interrogante $\dot{2}$ Es acaso suficiente la regulación normativa dada en el Perú para poder determinar la responsabilidad civil por daño ambiental? Como se pudo observar, existe regulación normativa, pero que carece de eficacia en el caso concreto.

Asimismo, es el Estado quien debe cumplir con la tarea de difundir conciencia ambiental para orientar la conducta humana hacia el respeto de los seres vivientes y no vivientes.

De lo expuesto cabe resaltar que si bien por un lado el Estado en sus tres niveles gubernamentales hoy en día otorga facultades a ciertas instituciones públicas, autónomas y que mediante los poderes de fiscalización y control, se dictan mecanismos de defensa en beneficio de las personas involucradas logrando así la interacción de los pobladores en pro de la defensa de sus derechos. Sin embargo, existe la otra cara 
de la moneda, donde muchas veces la población involucrada, hace poco o nada para, contrarrestar este problema de la contaminación ambiental. Esta situación se agrava por la falta de educación ambiental, servicios de salubridad y asesoría legal, es así que los gobiernos locales carecen de solvencia moral, por lo que se presume un problema de corrupción.

En suma, lo que se busca proteger es el medio ambiente, la persona y la propiedad como bienes jurídicos. En ese orden de ideas, se considera que la responsabilidad ambiental administrativa, no es suficiente para preservar la integridad de la persona, contrario sensu se determina la necesidad de petición de tutela jurisdiccional efectiva para la determinación de la "responsabilidad civil extracontractual" de las personas que se han visto afectadas en cuanto a su salud, patrimonio e integridad a consecuencia del hecho dañoso (quema de ladrillos).

\section{Análisis del Nexo Causal en el daño ambiental}

Como se pudo advertir anteriormente, uno de los presupuestos para la configuración de la responsabilidad civil es el nexo de causalidad, entendiendo a este como aquella actividad o conducta generadora del daño, ya que si ésta no se pudiese demostrar, no existiría acción resarcitoria por parte del agente contaminador a la víctima del daño ambiental.

Espinoza Espinoza (2011) señala respecto al nexo causal, que existe un análisis dual, es decir, se busca al sujeto responsable (causalidad de hecho) y hasta donde puede abarcar su responsabilidad (causalidad jurídica). Es por ello que en la doctrina se sostiene la importancia de determinar el nexo entre la conducta contaminante y el evento que constituiría la saturación ambiental.

Finalmente, se llega a la conclusión de que el déficit sobre la determinación del nexo de causalidad se da a raíz de la escasa actividad probatoria, es decir, cómo se demuestra que el agente ha cometido una conducta generadora de daño ambiental, una posible respuesta es considerar para estos casos la aplicación de la carga dinámica de la prueba, la cual permitirá que la carga probatoria recaiga sobre quien esté en mejor posición para asumirla.

\section{Factores de atribución}

El Artículo $144^{\circ}$ de la Ley General del Ambiente, prescribe que: "La responsabilidad derivada del uso o aprovechamiento de un bien ambientalmente riesgoso o peligroso, o del ejercicio de una actividad ambientalmente riesgosa o peligrosa, es objetiva". Esta responsabilidad obliga a reparar los daños ocasionados por el bien o actividad riesgosa, lo que conlleva a asumir los costos contemplados en el artículo $142^{\circ}$ de la norma precedente, y los que correspondan a una justa y equitativa indemnización; los de la recuperación del ambiente afectado, así como los de la ejecución de las medidas necesarias para mitigar los efectos del daño y evitar que éste se vuelva a producir".

Se percibió que existe también la posibilidad de daños por contaminación aun cuando no se haya infringido reglamento alguno: a pesar de adoptar las precauciones establecidas por el reglamento respectivo, los gases emitidos producto de la quema de insumos químicos para la cocción de ladrillos contamina el ambiente y producen la destrucción de la salud de las personas.

Si esta situación está regida por el artículo 1969 del Código Civil, los propietarios de las fábricas podrían demostrar que adoptaron todas las precauciones razonables y que, por tanto, no teniendo culpa, no recaería sobre ellos responsabilidad alguna. Sin embargo, se determinó que los propietarios de las fábricas deben ser considerados responsables objetivamente aun cuando no hayan sido negligentes porque, en muchos casos, los daños por contaminación son socialmente intolerables per se debido a la gravedad y a la amplitud del riesgo que crean; de tal manera, estos daños deben ser también incluidos en el campo de aplicación del artículo 1970 del Código Civil.

\section{Sujetos responsables}

No cabe duda que la legitimación pasiva recae en el causante del daño (persona natural o jurídica), pero qué pasa cuando son varias las personas responsables del mismo daño, es acaso una salida considerar la regla general de responsabilidades mancomunadas.

Es así que, la profesora Trujillo Moreno (2008, p. 215) formula que la mancomunidad es la menos satisfactoria para resolver los problemas que en materia de daños al medio ambiente se plantean porque dada la pluralidad de sujetos que se ven implicados, se fragmentaria la indemnización de un daño, que si bien ha podido ser causado por diversos sujetos, sólo tiene un único y final resultado, sin que éste pueda ser resarcido independientemente

Entonces es necesario considerar que los daños ambientales causados por varios agentes que actúan de forma independiente generan un daño final mayor al resultante de la suma por separado de algunas contribuciones dañosas. Por ello, en estos casos es preferible el uso de la responsabilidad solidaria que obligaría a los contaminadores a distribuir entre sí los daños causados. 
Algunas conclusiones son:

El fenómeno de contaminación suscitado en el Distrito de Huertas es un tema de suma importancia que involucra no solo a las entidades locales, sino que éste es de interés nacional e incluso internacional, siendo una de las soluciones de primer nivel el poder realizar una mejor regulación de la normativa nacional en cuanto a la responsabilidad civil que deriva del daño ambiental, ya que sería necesario regular dicha responsabilidad desde la perspectiva de los principios ambientales de precaución y prevención.

El Estado peruano tiene el deber de generar conciencia ambiental en los pobladores mediante campañas de concientización e implementación de curriculas escolares para mejorar la educación de los futuros ciudadanos sobre el tema.

\section{REFERENCIAS BIBLIOGRÁFICAS}

Andía Chávez, J. (2009). Manual de Derecho Ambiental: Doctrina, Jurisprudencia, Legislación. Lima: El Saber.

Benites, F. (26 de Marzo de 2013). Ladrilleras artesanales producen alta contaminación ambiental. Obtenido de Ladrilleras artesanales producen alta contaminación ambiental: http:// freundefbc.blogspot.pe/2013/03/ladrillerasartesanales-producen-alta.html

Betalleluz Montero, J., Gutierréz León, R., \& Mamani Romero, C. U. (20 de Abril de 2017). Entrevista a Juan sobre el uso de los combustibles usados en la quema de ladrillos. Disponible en: https://www. youtube.com/watch? $\mathrm{v}=\mathrm{NFOM9+YN80M}$

Betalleluz Montero, J., Gutierrez León, R., \& Mamani Romero, C. U. (20 de Abril de 2017). Entrevista sobre la contaminacion ambiental a la señora Timotea. Obtenido de https://www.youtube.com/ watch? $v=$ DqlQzIMpu50

Carhuatocto Sandoval, H. (2007). La Responsabilidad por Daño Aambiental Aplicada al Perú. IUS Doctrina \& Práctica, №4 Grijley, Lima, 193.

Daniel. (20 de Abril de 2017). Entrevista a trabajador de ladrillera de Condorcinja. (Robert, Entrevistador) Condorcinja, Jauja, Perú.

Del Castillo Gutiérrez, A. (28 de julio de 2017). Aplicacion del Derecho Civil a la Responsabilidad Ambiental. Disponible en: boletinderecho.upsjb. edu.pe/articulos/CIVIL_RESPONSABILIDAD.doc

Dirección General de Políticas, N. E. (31 de Mayo de 2010). MINAM. Obtenido de http://www. minam.gob.pe/wp-content/uploads/2013/10/ compendio_08_-_evaluacion_y_fiscalizacion_ ambiental_2.pdf

Distrito de Huertas. (15 de Junio de 2016). Obtenido de Distrito de Huertas: https://es.wikipedia.org/ wiki/Distrito_de_Huertas

Enperu. (24 de Junio de 2017). Obtenido de Enperu: http://www.enperu.org/junin/2017/01/23/ distrito-huertas-provincia-jauja-peru/

Espinoza Espinoza, J. (2011). Derecho de la Responsabilidad Civil. (6). Lima: Rodhas SAC

Gonzales, T. (20 de Abril de 2017). Entrevista sobre la contaminacion. (R. Guiterrez, Entrevistador)

Murillo, L. M. (30 de Octubre de 2013). Cultura ambiental: un estudio desde las dimensiones de valor, creencias, actitudes y comportamientos ambientales. (C. U. Adventista, Ed.) Colombia: Universidad Nacional de Colombia. Obtenido de file:///C:/Users/USUARIO/Downloads/DialnetCulturaAmbiental-5012134\%20(1).pdf

Taboada Cordova, L. (2000). "Responsabilidad Civil Extracontractual". Proyecto de Auto capacitación Asistida "Redes de Unidades Académicas Judiciales y Fiscales", Lima: Academia de la Magistratura

Trujillo Moreno, E., La protección jurídico privada del medio ambiente, 2008, p. 215. 